\title{
Temporal changes in the frequencies of the solar p-mode oscillations during solar cycle 23
}

\author{
E J Rhodes, Jr ${ }^{1,2}$, J Reiter ${ }^{3}$, J Schou ${ }^{4}, \mathbf{T}$ Larson $^{4}$, P Scherrer ${ }^{4}$, \\ J Brooks ${ }^{1}$, P McFaddin ${ }^{1}$, B Miller ${ }^{1}$, J Rodriguez $^{1}$, J Yoo $^{1}$ \\ ${ }^{1}$ Department of Physics \& Astronomy, University of Southern California, \\ Los Angeles, CA 90089-1342, U.S.A. \\ ${ }^{2}$ Space Physics Research Element, Jet Propulsion Laboratory, Pasadena, CA 91101, U.S.A. \\ ${ }^{3}$ Zentrum Mathematik, Technische Universität München, D-80333 Munich, Germany \\ ${ }^{4}$ HEPL 4085, Department of Physics, Stanford University, Stanford, CA 94305-4085, U.S.A.
}

\begin{abstract}
We present a study of the temporal changes in the sensitivities of the frequencies of the solar p-mode oscillations to corresponding changes in the levels of solar activity during Solar Cycle 23. From MDI and GONG++ full-disk Dopplergram three-day time series obtained between 1996 and 2008 we have computed a total of 221 sets of m-averaged power spectra for spherical harmonic degrees ranging up to 1000 . We have then fit these 284 sets of $m$-averaged power spectra using our WMLTP fitting code and both symmetric Lorentzian profiles for the peaks as well as the asymmetric profile of Nigam and Kosovichev to obtain 568 tables of p-mode parameters. We then inter-compared these 568 tables, and we performed linear regression analyses of the differences in p-mode frequencies, widths, amplitudes, and asymmetries as functions of the differences in as many as ten different solar activity indices. From the linear regression analyses that we performed on the frequency difference data sets, we have discovered a new signature of the frequency shifts of the p-modes. Specifically, we have discovered that the temporal shifts of the solar oscillation frequencies are positively correlated with the changes in solar activity below a limiting frequency. They then become anti-correlated with the changes in activity for a range of frequencies before once again becoming positively-correlated with the activity changes at very high frequencies. We have also discovered that the two frequencies where the sensitivities of the temporal frequency shifts change sign also change in phase with the average level of solar activity.
\end{abstract}

Keywords. Sun:P-mode, sun: magnetic field, sun: solar activity

\section{Introduction}

At the present time, there is still disagreement over the nature of the physical mechanism which causes the frequencies of the solar $p$-mode oscillations to change during each solar cycle. Our main motivation for this study was our desire to provide more-detailed evidence of the frequency dependence of the responses of the oscillation frequencies to changes in solar activity than has been available in the past. Our hope has been that we could provide additional constraints on the possible mechanism that causes these frequency shifts.

\section{Results}

From MDI and GONG++ full-disk Dopplergram three-day time series obtained between 1996 and 2008 we have computed a total of 284 sets of m-averaged power spectra 

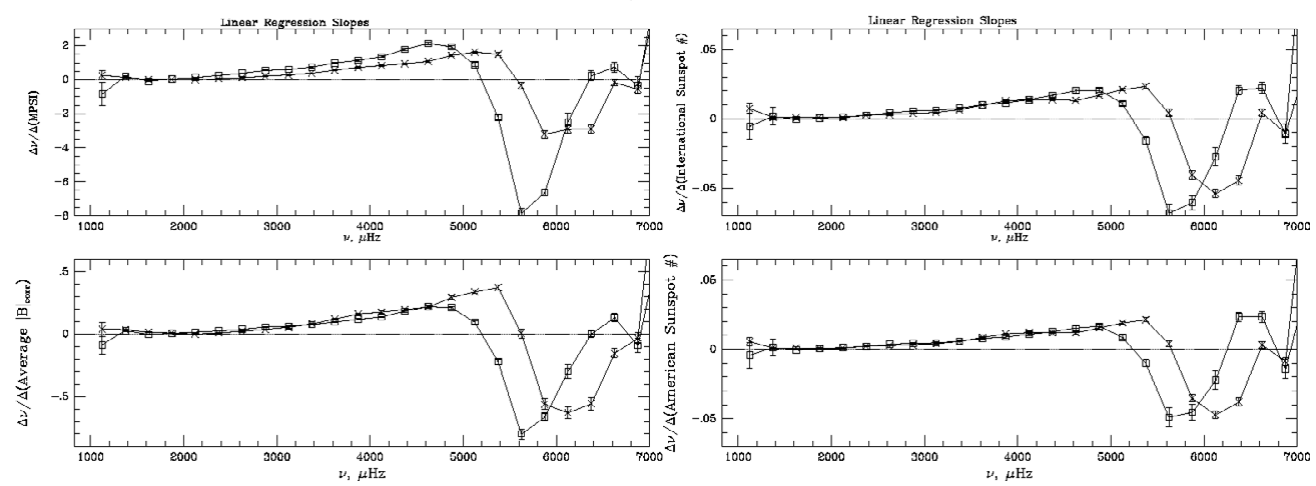

Figure 1. a.(top-left) The frequency dependence of the slopes of 25 separate pairs of linear regression analyses of binned symmetric-fit p-mode frequency shifts upon differences in Magnetic Plage Strength Index, MPSI. The $2001 \mathrm{GONG}+$ (solar maximum) results are shown as the Xs, while the 1996 MDI (solar minimum) results are shown as the squares. The \pm zero-crossing frequency increased systematically with increasing solar activity. Similarly, the $\mp$ zero-crossing frequency also increased systematically between 1996 and late 2001, as can be seen at the far right side of the panel. b.(lower-left) The frequency dependence of the 25 pairs of linear regression slopes for which changes in the average of the unsigned magnitude of the corrected photospheric magnetic field, $|B|_{\text {corr }}$, served as the independent variable. c.(top-right) Same as in panels a and b except that here differences in the three-day averages of the International Sunspot Number were used as the independent variable in the regression analyses. d.(bottom-right) In this case, differences in the three-day averages of the American Sunspot Number were used as the independent variable.

for spherical harmonic degrees ranging up to 1000. We have then fit these 284 sets of maveraged power spectra using our WMLTP fitting code and both symmetric Lorentzian profiles for the peaks as well as the asymmetric profile of Nigam and Kosovichev to obtain 568 tables of p-mode parameters. We then inter-compared these 568 tables, and we performed linear regression analyses of the differences in p-mode frequencies, widths, amplitudes, and asymmetries as functions of the differences in as many as ten different solar activity indices. Examples of the frequency dependence of some of the slopes of these linear regression comparisons are shown here in Figure 1. In Figure 1a, we show the frequency dependence of the 25 different slopes which we obtained from the linear regression analyses of the GONG++ frequency shifts upon the differences in the Magnetic Plage Strength Indicator (MPSI) as the Xs, while the 25 corresponding MDI regression slopes are shown as the squares. In this panel we can see that the regression slopes did in fact change sign at two different frequencies in the case of the GONG++ and MDI analyses. Based upon these results, we have defined the frequency at which the sign of the regression slopes change from being positive to being negative as the \pm zero-crossing frequency. Figure 1a also shows that both sets of regression slopes later changed sign again from negative to positive at much higher frequencies. We refer to the locations of these changes in sign as the $\mp$ zero-crossing frequencies. In fact, both the \pm and the $\mp$ zero-crossing frequencies were shifted toward higher values for the GONG++ observations from solar maximum in comparison with the zero-crossing frequencies of the MDI observations from solar minimum conditions. We believe that the most intriguing new feature that can be seen in Figure 1a is the obvious displacement of the solar maximum curve (i.e., the $\mathrm{Xs}$ ) toward the right side of the panel. The location where the frequency shifts change sign from positive to negative was moved to a 
higher frequency when the level of solar activity increased from the minimum levels of 1996 to the maximum levels of late-2001. To our knowledge, this frequency shift in the zero-crossing frequency of the temporal frequency shifts has never been seen before by any other helioseismology studies. Not only is the zero-crossing frequency shifted toward higher frequencies at the time of solar maximum, but the entire high-frequency portion of the GONG++ curve is shifted toward the right side of the plot. The same behavior can be seen in Figure 1b where the independent variable used in the regression analyses was the difference in average value of the unsigned magnitude of the corrected photospheric magnetic field, $|B|_{\text {corr }}$. The same behavior can also be seen in Figures $1 \mathrm{c}$ and 1d, where we have plotted the regression slopes which we obtained when we employed the differences in the International and American Sunspot Numbers as the independent variables, respectively.

The dependence of the 19 mean \pm zero-crossing frequencies upon the mean levels of the International Sunspot Number (ISN) are shown as the set of Xs in Figure 2a. There is a clear tendency for the zero-crossing frequencies to increase as the mean value of the ISN increases. In fact, the lower solid line shown in Figure $2 \mathrm{a}$ is the linear regression fit to all 19 of the \pm zero-crossing frequencies. We also show the dependence of the 18 mean $\mp$ zero-crossing frequencies upon the mean levels of the ISN as the set of circles in Figure 2a. We have included the linear regression fit to those $18 \mp$ zero-crossing frequencies as the upper solid line. The similarity of the two linear regression fits show that both the \pm and the $\mp$ zero-crossing frequencies were positively correlated with the changes in the mean level of solar activity during each time interval. In Figure $2 \mathrm{~b}$ we present similar results to those shown in Figure 2a except that the asymmetric fitting profiles were used instead of the symmetric Lorentzian profiles. The $\mp$ zero-crossing frequencies and regression line are very similar to the results obtained using the symmetric profiles. However, the 19 \pm zero-crossing frequencies computed using the asymmetric profiles are systematically lower than the corresponding points in Figure 2a.

\section{Conclusion}

After discovering some of the basic behaviors of zero-crossing frequencies in recent years, we have now been able to track the way that these frequencies change on a yearly basis from 1996 to 2008 . We have been pleasantly surprised to find that the newest points on our graph exhibit a linear relationship between the zero-crossing frequency and average solar activity. We believe that this change in the location of the zero-crossing frequency can be used as a new tool for probing changes in the sub-surface shear layer since some of the previous theoretical models which have been developed to explain the temporal frequency shifts have found that various combinations of temperature and magnetic field changes can result in a change in the sign of the frequency shifts at high frequencies (Jain \& Roberts, 1993; Jain, 1995; Johnston et al., 1995). Hence, we believe that this new observational signature will allow estimates to be made of the changes in both temperature and field strength which occurred between 1996 and 2001. To summarize this important set of results, we have demonstrated

- that the p-mode oscillations demonstrate a complicated pattern in which their frequencies are correlated with changes in solar activity at low frequencies and then become anti-correlated with those changes in activity at intermediate frequencies before once again being positively correlated with the changes in activity at the highest frequencies;

- that the frequencies where the frequency shifts change from being correlated to being anti-correlated with solar activity change with the mean level of activity; 


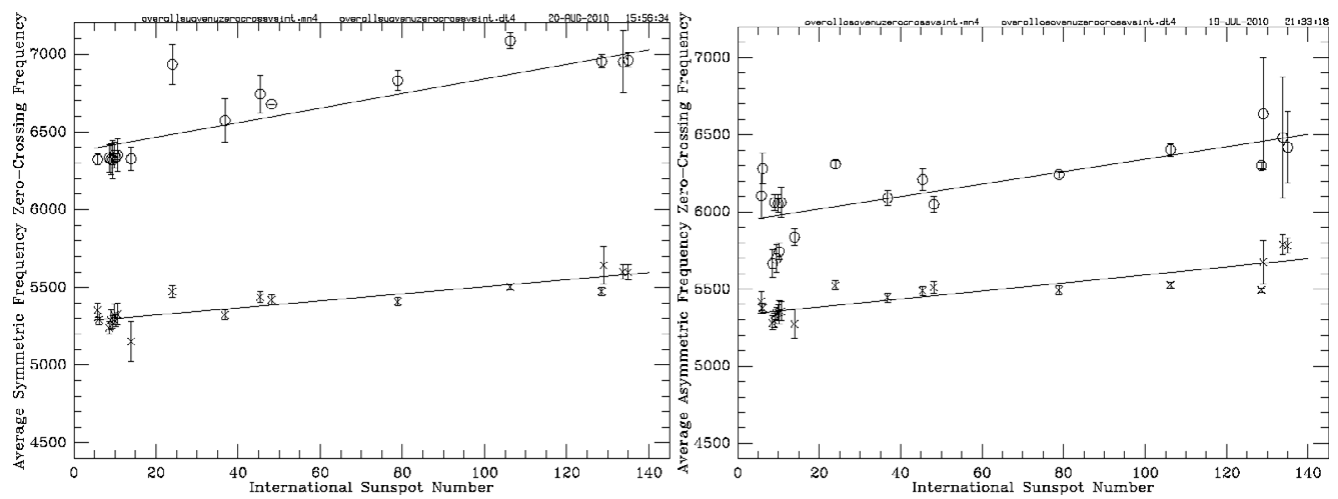

Figure 2. a.(left) Dependence of the mean \pm zero-crossing frequencies (the Xs) for all 19 different time intervals upon the mean value of the ISN during each of those 19 time periods. The straight line is the linear regression fit to the 19 data points. It shows that the differences in the zero-crossing frequencies which were evident in Figure 1 were the extreme cases of a solar cycle dependence of those zero-crossing frequencies. The 18 corresponding mean $\mp$ zero-crossing frequencies are shown as the 18 open circles in the upper portion of the panel. The linear regression fit to the 18 mean $\mp$ zero-crossing frequencies is shown as the upper solid line. b.(right) Similar results to those shown in the left-hand panel except that the asymmetric fitting profiles were used instead of the symmetric Lorentzian profiles. The \pm zero-crossing frequencies and regression line were very similar to the results obtained using the symmetric profiles. However, the 19 ₹ zero-crossing frequencies computed using the asymmetric profiles were systematically lower than the corresponding points in panel a.

- that the frequencies at which the frequency shifts go back to being positively correlated with the activity changes also changes with the mean level of activity;

Taken together, all of these new signatures of the temporal shifts in the p-mode frequencies should give the theorists new information to include in their refined models of the causes of these temporal frequency shifts.

\section{Acknowledgements}

In this work we utilized data from the Michelson Doppler Imager (MDI) on the Solar and Heliospheric Observatory (SOHO). SOHO is a project of international cooperation between ESA and NASA. The Stanford component of this work was supported by NASA Awards NNX07AK36G and predecessors and NAS5-02139. The portion of this research which was conducted at USC and at the Technical University of Munich was supported by the following grants to USC: NASA Grants NNX08AJ24G, NNX06AC24G, NAG5-13510, NAG5-11582, and NAG5-11001, NSF Grant AST-0307934, and by the following sub-awards from Stanford University to USC: Number 1503169-33789-A and Number 14405890-26967. Some of the computations for this work were carried out at USC using the HPCC and some of them were carried out with the use of the JPL Origin 2000 supercomputers. This work utilizes data obtained by the Global Oscillation Network Group (GONG) project, managed by the National Solar Observatory, which is operated by AURA, Inc. under a cooperative agreement with the National Science Foundation. The data were acquired by instruments operated by the Big Bear Solar Observatory, High Altitude Observatory, Learmonth Solar Observatory, Udaipur Solar Observatory, Instituto de Astrofisico de Canarias, and Cerro Tololo Interamerican Observatory. 


\section{References}

Jain R., 1995, ESA SP-376, 69

Jain R. \& Roberts B., 1993, Astrophys. J. 414, 898

Johnston A., Roberts B., \& Wright A. N., 1995, ASP Conf. Ser., 76, 264 